\title{
Interactive Technology Solutions for Prisoners: An Environment where Sub-optimal User Interfaces can have Significant Impact?
}

\author{
Kyle Boyd \\ Ulster University \\ Belfast, BT15 1ED \\ ka.boyd@ulster.ac.uk
}

\author{
Raymond Bond \\ Ulster University \\ Jordanstown, BT37 0QB \\ rb.bond@ulster.ac.uk
}

\author{
Emer O'Kane \\ Core Systems NI LTD \\ Belfast, BT14 8AD \\ emer@coresystems.biz
}

\begin{abstract}
As a work in progress, we are currently investigating if current offender technology used in prisons worldwide meets the needs of prisoners who have low computer and reading literacy. In addition to the obvious requirements of a prisoner's persona, researchers have identified that emotions in prisons can be heightened and usability issues with technology can be disruptive and result in unwanted behavior. With this in mind, we aim to evaluate the user interfaces using validated usability testing protocols.
\end{abstract}

Prisoners, Offender Technology, User Interfaces, User Experience, Usability Testing, Human-Computer Interaction

\section{INTRODUCTION}

Over the last decade, the Internet, technology and computing have changed the way society conducts business, engages with politics and digests entertainment through interactive devices such as smart phones, tablets and now wearable devices. Indeed technology can be seen as a way to help those in need but it can also be used to make tasks more efficient and in this case, does so for prisoners and prison officers.

Currently in the UK there are 85,641 prisoners in custody [1]. Studies suggest that between $22 \%$ and $47 \%$ of those prisoners do not have any formal qualifications [2], [3]. Furthermore, between 20$30 \%$ of prisoners have learning difficulties that affect their ability to cope within criminal justice system. Indeed, it is estimated by the PRT that $60 \%$ of prisoners have a reading ability equivalent or less than that of a five year old child and around $40 \%$ of prisoners need specialist support for dyslexia [4]. These figures contradict a little with this: $46 \%$ of people entering the prison system have literacy skills no higher than those broadly expected of an 11 year old child[5].

In the UK up to $60 \%$ of a prison officer's time at work can involve dealing with information requests from prisoners and in turn undertaking paper-based administration. Significant efficiency savings can be made using interactive technology and effective user interfaces to deliver prisoner services such as self-selected food ordering, book loans, registration for educational courses and entertainment (refer to Figure 1).

In order to move prisons from a bureaucratic, paper-based we must introduce change; towards integrated digital services that encourage a new kind of interaction with prisoners; empowering them to do things for themselves, to become more actively involved in their rehabilitation and to develop life skills that will benefit them on their return to their community [6].

Nevertheless, given emotions in prison are a key concern, sub-optimal usability of user interfaces can raise levels of frustration for the prisoner which can escalate to inappropriate behaviour and disruption [7], [8]. Up to $47 \%$ of the current prison population in the UK do not have any qualifications suggesting low literacy levels [2]. There is research, which considers designing interfaces for those with low literacy [9], [10]. This is something Direct2inmate technology aims to implement and test by conducting a usability experiment with prisoners who have never used the technology to see if usability standards are met.

\section{DIRECT2INMATE TECHNOLOGY}

The interactive prisoner technology called Direct2inmate is a secure platform for prisoners to securely access information and services for themselves. It provides tools for prisoners to 
rehabilitate and successfully re-enter society through self-motivation.

The platform supports applications to provide prisoners with services such as electronic More specifically, we aim to evaluate if user interaction issues are hindering the potential for optimal use with the system.

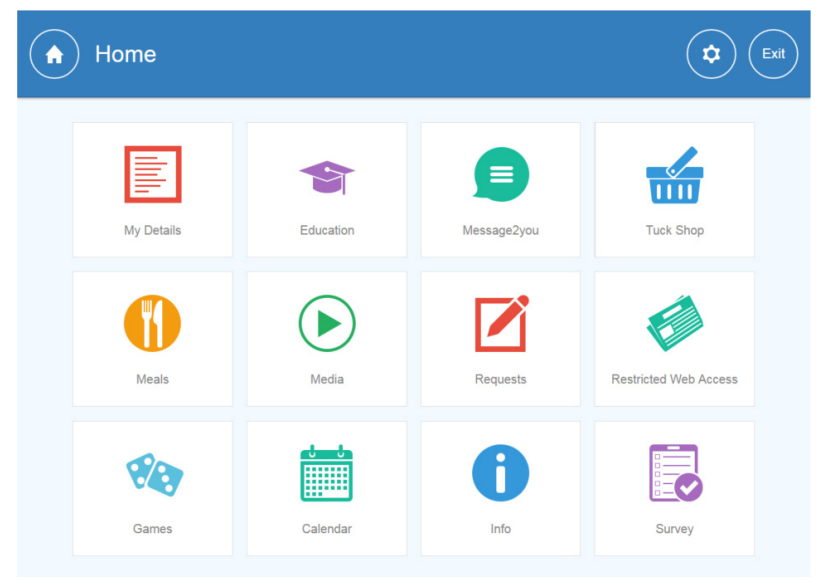

Figure 1: An example of the Direct 2 Inmate which will be usability tested.

\section{USABILITY TESTING OF INMATE TECHNOLOGY}

There are a number of approaches that can be used to conduct a Usability Test. The most common approach is the 'Think Aloud' protocol where a participant verbalises their cognitive process while they are completing a series of tasks. This helps demonstrate and highlight the usability issues being encountered as they interact with the system. The advantage of the Think Aloud protocol is that it offers a rapid approach to conducting and obtaining first hand insight into the thought processes associated with different tasks [8]. To date this approach has been used during the design and implementation phases of software development projects; however, it can also be used for evaluation purposes, which will be its primary focus in this project. The advantage of the Think Aloud protocol is that it offers a rapid approach to conducting and obtaining first hand insight into the thought processes associated with different tasks [11]. To date this approach has been used during the design and implementation phases of software development projects; however, it can also be used for evaluation purposes, which will be its primary focus in this project.

Using a Tobii Eye Tracker [12], the participants eye-gaze and scan-path information will also be non-invasively recorded while they interact with the prisoner technology solution. The Tobii eye tracking and eye control technology makes it possible for computers to know exactly where participants are looking on screen using infrared light which is used messaging, submitting requests/forms, e-learning and shop ordering. It is our intention to consider these interactive prisoner technology solutions to test if they meet the needs of prisoners.

to reflect of the cornea and in tune using trigonometric functions to approximate eye fixations. Users are not required to wear additional devices since it is non-intrusive and the device is simply placed underneath the monitor. Mouse movements and audio will also be recorded.

Before and after each task is completed, the single ease question (SEQ) [13] will be asked, which is a 7-point rating scale to assess how difficult users find a task. It's administered before and immediately after a user attempts a task, which indicates if the system met the user's expectation. After the usability test is completed, each participant will complete a post test usability questionnaire which will be scored using the Systematic Usability Scale (SUS) [14]. SUS provides a reliable tool for measuring usability. It consists of a 10 item questionnaire with a five-scale Likert style response ranging from strongly agree to strongly disagree. After completion a universal SUS score is given.

Prior to the test, each participant will also be provided with a pre-test questionnaire to collect information in relation to participant background information such as age and their level of experience using the Internet. Following the usability test, participants will be provided with a post-test questionnaire and from this data it will be possible to identify a SUS score and which elements of the interface they liked and which problems they encountered and how they would like these to be addressed. Participants will be encouraged to express their opinion and thoughts throughout the usability test. Notes taken, eye tracking recordings and audio recordings will be analysed to assess the following parameters:

i Time spent to accomplish each task (task completion times)

ii Frequency and severity of problems and usability errors participants encountered.

iii Successfully accomplished tasks (task completion rate)

iv Un-successful task attempts (task failure rate)

For quantitative analysis, we will use averages (mean and median) and standard deviation and inter-quartile range. A hypothesis test such as a ttest will be used to test the differences between the pre-task and post-task single ease question scores which will highlight if any of the tasks did or did not meet the user's expectation. 


\section{FUTURE WORK}

Ethical approval for the study has been granted by Research Institute of Art and Design at Ulster University. Recruitment is being conducted by Core Systems - the developers of Direct2inmate technology. Data collection and analysis is currently underway.

\section{REFERENCES}

[1] "Prison population figures: 2015 - Publications GOV.UK." [Online]. Available: https://www.gov. uk/government/statistics/prison-populationfigures-2015. [Accessed: 29-Apr-2016].

[2] P. R. Trust, "Prison: the Facts," 2013. [Online]. Available: http://www.prisonreformtrust.org.uk/ Portals/0/Documents/Prisonthefacts.pdf. [Accessed: 29-Apr-2016].

[3] UK Gov, "No Title," Prior Qualifications of Adult OLASS learners, 2015. [Online]. Available: https://www.gov.uk/government/ uploads/system/uploads/attachment_data/file/ 489417/bis-16-31-prior-qualifications-adultolass-learners-2015.pdf. [Accessed: 05-May2016].

[4] "No One Knows - offenders with learning difficulties and learning disabilities." [Online]. Available: http://www.prisonreformtrust.org.uk/ uploads/documents/noknl.pdf. [Accessed: 29Apr-2016].

[5] C. Taylor, "Prisoner education in the UK: a review of the evidence by Prisoners' Education Trust," Prison Serv. Journal, no. 223, pp. 44$51,2016$.
[6] R. Metzner, "Through the Gateway: How Computers Can Transform Rehabilitation," 2013.

[7] V. Knight, "Modus Vivendi: The cell, emotions, social relations and television," pp. 1-19, 2015.

[8] A. Bruun, E. L.-C. Law, M. Heintz, and L. H. A. Alkly, "Understanding the Relationship Between Frustration and the Severity of Usability Problems: What Can Psychophysiological Data (Not) Tell Us?," in Proceedings of the $2016 \mathrm{CHI}$ Conference on Human Factors in Computing Systems, 2016, pp. 3975-3987.

[9] B. M. Chaudry, K. H. Connelly, K. A. Siek, and J. L. Welch, "Mobile interface design for lowliteracy populations," 2nd ACM SIGHIT Int. Heal. informatics Symp., pp. 91-100, 2012.

[10] N. Ahmad, U. Shoaib, and P. Prinetto, "Usability of Online Assistance From Semiliterate Users' Perspective," Int. J. Hum. Comput. Interact., vol. 31 , no. 1, pp. 55-64, 2015.

[11] S. Krug, Don't Make Me Think! A Common Sense Approach to Web Usability, vol. Second Edi. Berkley: Newriders, 2009.

[12] T. E. Research, "No Title." [Online]. Available: http://tinyurl.com/726sugb.

[13] J. Sauro and J. R. Lewis, Chapter 2 Quantifying User Research, vol. 11. 2012.

[14] J. Brooke, "SUS - A quick and dirty usability scale," Usability Eval. Ind., vol. 189, no. 194, pp. 4-7, 1996. 